\title{
Sensor Fusion Based Obstacle Detection/Classification for Active Pedestrian Protection System
}

\author{
Ho Gi Jung ${ }^{1,2}$, Yun Hee Lee ${ }^{1}$, Pal Joo Yoon ${ }^{1}$, In Yong Hwang ${ }^{1}$, and Jaihie Kim ${ }^{2}$ \\ ${ }^{1}$ MANDO Corporation Central R\&D Center \\ 413-5, Gomae-Dong, Giheung-Gu, Yongin-Si, Kyonggi-Do 446-901, Republic of Korea \\ \{hgjung, p13468, pjyoon, iyhwang\} @mando.com \\ ${ }^{2}$ Yonsei University, School of Electrical and Electronic Engineering \\ 134, Sinchon-Dong, Seodaemun-Gu, Seoul 120-749, Republic of Korea \\ \{hgjung, jhkim\} @yonsei.ac.kr
}

\begin{abstract}
This paper proposes a sensor fusion based obstacle detection/classification system for active pedestrian protection system. At the frontend of vehicle, one laser scanner and one camera is installed. Clustering and tracking of range data from laser scanner generate obstacle candidates. Vision system classifies the candidates into three categories: pedestrian, vehicle, and other. Gabor filter bank extracts the feature vector of candidate image. The obstacle classification is implemented by combining two classifiers with the same architecture: support vector machine for pedestrian and vehicle. Obstacle detection system recognizing the class can actively protect pedestrian while reducing false positive rate.
\end{abstract}

\section{Introduction}

There are two explicit trends of $\mathrm{CW}$ (Collision Warning)/CA(Collision Avoidance) development: driver behavior model [1][2][3] and pedestrian protection [4][5]. Because these factors use the information of driving situation to reduce annoying false positive action while achieving fast response and reliable operation, they could be understood from the viewpoint of situation awareness. Driver monitoring system observes driver and measures the probability of whether driver perceive upcoming dangerous situation or not. Driver's perception status is used to modify the risk assessment estimated by time to collision [2]. Driver behavior model can modify the driving situation criticality level by considering the potential risk of collision and the adequacy of the driver's behavior [3]. Pedestrian protection system, which protects vulnerable road user, i.e. pedestrian, from traffic accident, is thought to be the most efficient and important method to reduce the fatalities of traffic accident [4]. Intelligent night vision is a kind of pedestrian protection system providing enhanced forward image in nighttime and recognizing pedestrian automatically [5]. Therefore, next generation CW/CA system is expect to not only detect forward obstacle but also recognize its class. Obstacle classification makes it possible for CW/CA system to consider the kind of obstacle. Especially, active safety system is expected to protect pedestrian more actively. This paper is related with forward obstacle detection and classification. 
Vehicle detection has been one of the most important sensing problems in active safety system and driver assistance system. Sun's recent survey successfully summarizes up-to-date research activities on vehicle detection [6]. He analyzes vehicle detection process into two phases: HG(Hypothesis Generation) and HV(Hypothesis Verification). For the hypothesis generation phase, three kinds of methods are listed: knowledge based methods, stereo based methods, and motion based method. For the hypothesis verification phase, two kinds of methods are listed: template based method, and appearance based method. Recently, almost vehicle detection systems adopt the appearance based method, which classifies the hypotheses into 'vehicle' class or 'non-vehicle' class. Among them, SVM(Support Vector Machine) with Gabor filter is reported to produce the best performance [7].

Comparatively, pedestrian detection has shorter history, but is supposed to be the most critical technology for the reduction of traffic accident fatality [8]. Pedestrian detection method can be classified into three categories: range sensor based methods, vision based methods, and sensor fusion based methods.

Pedestrian detection with range sensor detects clusters of neighboring range data, then determines whether the clusters are corresponding to 'pedestrian' or not based on several kinds of attributes. Motion and width of cluster tracked temporally can be fed into pattern classifier [9]. With mm-wave radar, relation between range distribution and speed distribution is used for obstacle classification [10]. In other words, pedestrian has little range distribution, but large speed distribution compared to vehicle and stationary object.

Vision based pedestrian can be classified into three categories again: rhythmic motion based method, contour matching based method, and appearance based method. Cristóbal Curio verifies the candidates by checking the periodic motion caused from gait [11]. D. M. Gavrila developed contour matching based pedestrian detection. His chamfer system proposed a tree structure of pedestrian contours and distance transform based matching [12]. Appearance based pedestrian detection is similar to the appearance based vehicle detection. A. Broggi used morphological characteristics and the strong vertical symmetry of the human shape [13]. Liang Zhao used stereo based segmentation and neural network based recognition [14]. As proved in vehicle detection, SVM is supposed to be the best classifier also in pedestrian detection. Stereo based segmentation, edge features, and SVM classifier showed good classification performance and efficient computation possibility [15]. As in vehicle detection case, wavelet feature and SVM classifier seem to produce the best performance and robustness [16][17].

Sensor fusion based methods focus on the reduction of HG workload and the increase of hypothesis reliability. Therefore, they generally select sequential data fusion method [18]. Range sensor efficiently narrows the search range, although it is not complete, such that real time implementation becomes possible. Actually, to some extent, sensor redundancy is required to meet the reliability needs in automotive field [19].

As shown in Fig. 1, we select sequential sensor fusion based method to meet real time requirement. Laser scanner provides range data including true 'vehicle' and 'pedestrian', which are confused with noise. Range data clustering and tracking find potential obstacle eliminating noise to some extent. Two pattern classifiers are designed: one for vehicle detection, and the other for pedestrian detection. These pattern 


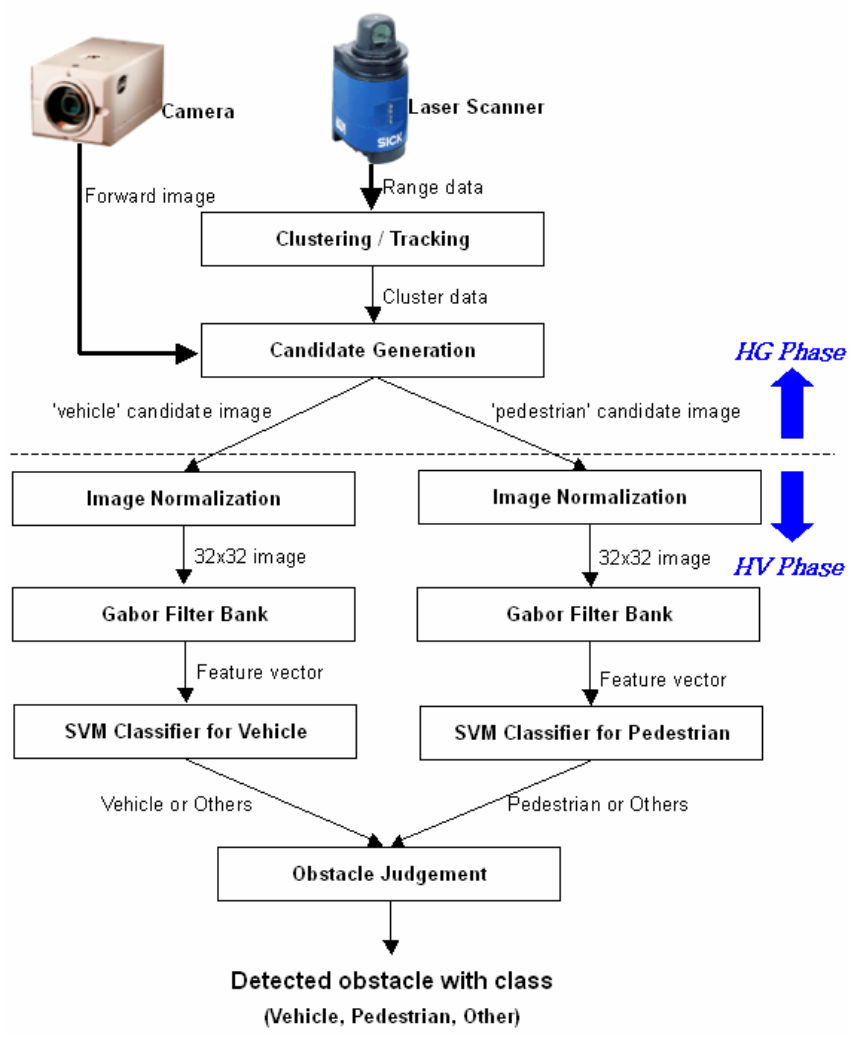

Fig. 1. Architecture of proposed sensor fusion based obstacle detection/classification system

classifiers are 'two class problem' solvers, i.e. true or not problem. Each classifier is implemented by SVM with Gabor filter bank, which is proved successful in various applications. At the decision phase, the outputs of two classifiers are simply integrated to determine the class of obstacle. In ambiguous situation, when vehicle classifier and pedestrian classifier output positive result simultaneously, the pedestrian classifier is selected to protect pedestrian actively. In this case, the system should accept the increase of false alarm rate. However, it can be justified by the fact that total error rate is sufficiently low and accident with pedestrian causes a fatal result. 'Other' class means a false obstacle caused by laser scanner noise or roadside object. Experiments show that proposed system can detect and classify obstacles successfully. Because Gabor filter bank - SVM is suitable for hardware implementation and the same architecture is used for two obstacle classes, our proposed system is expected to be a practical solution for mass production.

\section{Candidate Generation}

Laser scanner outputs range data, which are measured distance to objects with respect to azimuth angle. Objects can be identified by detecting clusters in the range data. 
Fig. 2(b) shows the result of clustering adjacent data points with pre-defined distance, e.g. $0.3 \mathrm{~m}$. Detected clusters are tracked by Kalman filter to ensure the robustness of cluster detection. Assigned individual identification enables temporal signal processing. Distance, azimuth angle, changing rate of distance, changing rate of azimuth angle and cluster width are used as the state variables of Kalman filter.

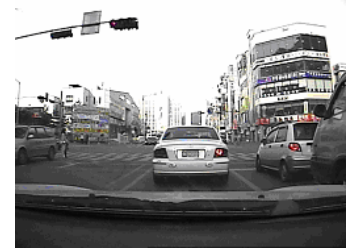

(a) Forward image from camera

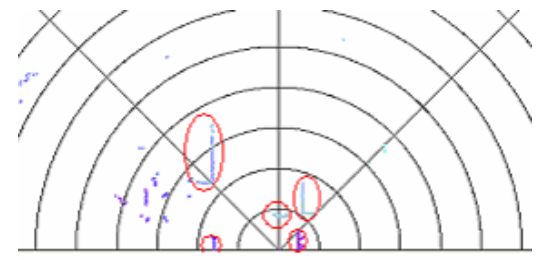

(b) Range data from laser scanner and clusters

Fig. 2. Forward image and range data with detected clusters

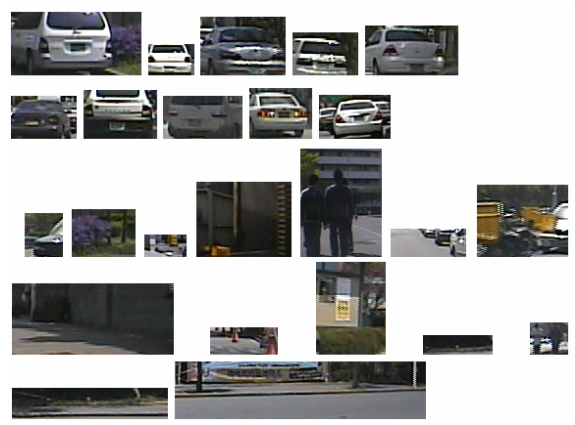

(a) 'vehicle' candidate images



(b) 'pedestrian' candidate images

Fig. 3. Generated candidate images. In 'vehicle' candidate images, there are correct and incorrect cases. In 'pedestrian' candidate images, there are correct and incorrect cases.

Candidate image corresponding to each tracked cluster is extracted. Polar coordinate $(r, \theta)$ of recognized cluster can be converted easily to world coordinate $\boldsymbol{P}_{W}\left(X_{W}\right.$, $\left.Y_{W}, Z_{W}\right) . Z_{W}$ plane is assumed to be the ground surface. Image coordinate $\boldsymbol{P}_{\boldsymbol{I}}\left(X_{I}, Y_{I}\right)$ corresponding to the world coordinate $\boldsymbol{P}_{\boldsymbol{W}}$ can be derived by perspective projection model. An image portion with the width of corresponding cluster and specific height is extracted as a candidate image. The specific height is $1.5 \mathrm{~m}$ for vehicle and $2 \mathrm{~m}$ for pedestrian. Therefore, one vehicle candidate image and one pedestrian candidate image are created for each cluster. Since each cluster creates two candidate images with two specific heights, vehicles or pedestrians with unusual height may hinder the following pattern recognition. However, the alignment of an obstacle may be maintained constantly since the height of extracted candidate image is determined from the ground surface. Fig. 3 shows the example of generated obstacle candidate image. Incorrect cases are caused by street side objects and laser scanner noise. The roles of classifiers are determining whether the candidate is correct or not. 
Since segmented candidate images have various size and shape depending on width and distance, the images are required to be converted into uniform size. Both vehicle recognition module and pedestrian recognition module use 32x32 image as input. Fig. 4 illustrates the result of 'vehicle' candidate image normalization and 'pedestrian' candidate image normalization.
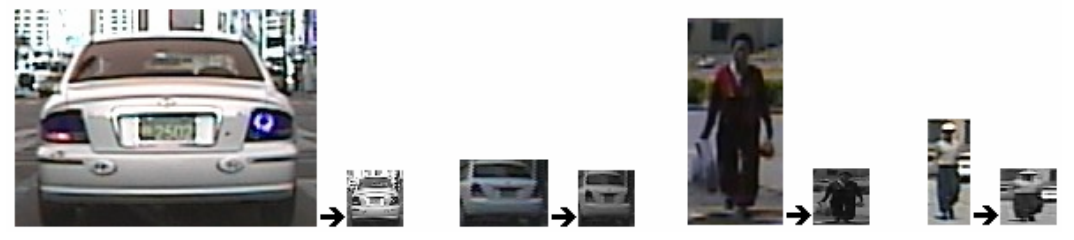

Fig. 4. Candidate image normalization procedure

\section{Feature Vector Construction}

Gabor filter is product of two-dimensional Gaussian function and sine wave [20]. Gabor filter can measure the image power of a specific frequency and direction at a specific location. $\sigma_{x}$ and $\sigma_{y}$ represent standard deviation of x-axis and y-axis. $W$ is the frequency of spatial wave. Direction of sine wave is determined by rotating axis by $\theta$. The definition of Gabor filter in frequency domain is shown in equation (2).

$$
\begin{gathered}
g(x, y)=\left(\frac{1}{2 \pi \sigma_{x} \sigma_{y}}\right) \exp \left[-\frac{1}{2}\left(\frac{x^{2}}{\sigma_{x}^{2}}+\frac{y^{2}}{\sigma_{y}{ }^{2}}\right)+2 \pi j W x\right] \\
G(u, v)=\exp \left[-\frac{1}{2}\left(\frac{(u-W)^{2}}{\sigma_{u}{ }^{2}}+\frac{v^{2}}{\sigma_{v}{ }^{2}}\right)\right]
\end{gathered}
$$

Gabor filter bank is a group of Gabor filters with various shapes in a frequency domain. Since Gabor filter functions as band-pass filter for different frequencies, it is commonly used to create feature vector of images. We follow Manjunath's approach: equally dividing frequency space in phase angle and logarithmically dividing frequency space in magnitude direction [21].

Every filter is designed for its half height boundary to meet neighboring filters' boundaries. Our system uses 4 scales and 6 orientations: $S$ (scale number) $=4$, $K($ orientation number $)=6$. Once $\mathrm{u}$-axis directional standard deviation $\sigma_{u}$ can be derived for a Gabor filter located on $\mathrm{u}$-axis, other cases can be derived by rotating the result. In u-axis direction, average value and standard deviation of sth Gaussian function located on u-axis, $G_{s}$, are in the manner of geometrical progression with the average value and standard deviation of the first Gaussian function as shown in equation (3). $U_{l}$ represents the lowest frequency, and $U_{h}$ represents the highest frequency. Multiplication factor in logarithmic scale, $a$, is defined as in equation (4), which represents the frequency ratio of Gabor filters consecutive in scale. 


$$
\begin{gathered}
G_{s}(u) \sim N\left(U_{s}, \sigma_{u}\right)=N\left(U_{l} a^{s-1}, \sigma_{0} a^{s-1}\right) \\
a=\left(\frac{U_{h}}{U_{l}}\right)^{\frac{1}{S-1}} \quad \text { or } \quad \ln a=\frac{1}{S-1}\left(\ln U_{h}-\ln U_{l}\right)
\end{gathered}
$$

Average value of $s$ th Gaussian function located on u-axis, $U_{s}$, is defined in equation (5). Its u-axis standard deviation, $\sigma_{u}$, and v-axis standard deviation, $\sigma_{v}$, is defined in equation (6) and (7) respectively [21].

$$
\begin{gathered}
U_{s}=U_{l} \cdot a^{s-1} \\
\sigma_{u}=\frac{(a-1) U_{s}}{(a+1) \sqrt{2 \ln 2}} \\
\sigma_{v}=\tan \left(\frac{\pi}{2 K}\right) \frac{\left(U_{s}-2 \ln 2 \frac{\sigma_{u}{ }^{2}}{U_{s}}\right)}{\sqrt{2 \ln 2-(2 \ln 2)^{2} \frac{\sigma_{u}^{2}}{U_{s}^{2}}}}
\end{gathered}
$$

The direction of 2-dimensional wave is set by substituting $(x, y)$ by $\left(x^{\prime}, y^{\prime}\right)$, which is rotated to the $k$ th section of $K$ equal angular sections like equation (8). Especially, Gabor wavelet is created by multiplying the magnitude with $a^{-s}$ to divide the power of high-pass filter into two child-filters recursively. Figure 5 shows the designed 24 Gabor filters in frequency domain. It is learned that because the adjacent Gabor filters contact each other at half height to minimize the information overlapping, each of designed filters functions as a band-pass filter for a specific frequency region [21].

In order to overcome segmentation deviation, only the statistical characteristic of Gabor filtering results of superposed sub-windows are used for the recognition process. Features are extracted for 9 overlapped sub-windows of candidate image. Candidate image with $32 \times 32$ size is divided into 9 sub-windows with $16 \times 16$ size. Each $16 \times 16$ sub-window overlaps a half of each other. Therefore, even if there is alignment deviation after the creation of candidate images, the extracted feature will bring similar results. The feature vector for recognition consists of mean, standard deviation, and skewness of convolutions between 9 sub-windows and 24 Gabor filters. Therefore, the dimension of feature vector is $648(=9 \times 24 \times 3)$.

$$
g_{s}(x, y)=a^{-s}\left(\frac{1}{2 \pi \sigma_{x} \sigma_{y}}\right) \exp \left[-\frac{1}{2}\left(\frac{x^{\prime 2}}{\sigma_{x}^{2}}+\frac{y^{\prime 2}}{\sigma_{y}^{2}}\right)+2 \pi j U_{s} x^{\prime}\right]
$$




$$
\text { where, }\left\{\begin{array}{l}
x^{\prime}=x \cos \left(\frac{k \pi}{K}\right)+y \sin \left(\frac{k \pi}{K}\right) \\
y^{\prime}=-x \sin \left(\frac{k \pi}{K}\right)+y \cos \left(\frac{k \pi}{K}\right), \sigma_{x}=\frac{1}{2 \pi \sigma_{u}}, \sigma_{y}=\frac{1}{2 \pi \sigma_{v}}
\end{array}\right.
$$

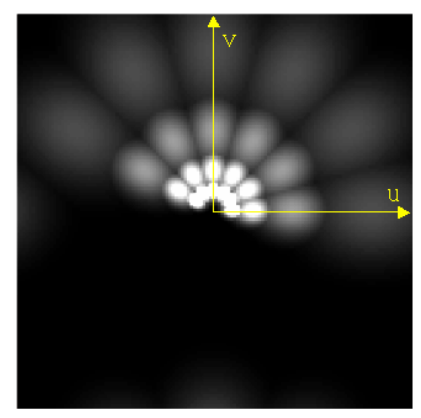

Fig. 5. Designed Gabor filter bank. 24 Gabor filters are overlapped in frequency domain.

\section{Support Vector Machine Based Classification}

SVM is a kind of classification method with hyperplane [22]. Decision boundary is defined by a $N$-dimensional vectors $\mathbf{w}$ and $b$ defining a hyperplane as shown in equation (9). In the case of two-class problem, the decision function is defined by the sign of the projection of input vector $\mathbf{x}$ on the hyperplane as shown in equation (10).

$$
\begin{gathered}
(\mathbf{w} \cdot \mathbf{x})+b=0 \quad, \text { where } \mathbf{w} \in \mathcal{R}^{N}, b \in \mathcal{R} \\
f(\mathbf{x})=\operatorname{sign}((\mathbf{w} \cdot \mathbf{x})+b)
\end{gathered}
$$

SVM assumes the maximum separating margin between two classes is the optimal condition. There are many hyperplanes dividing two classes. An optimal hyperplane should be designed as far as possible from two classes simultaneously. If margin $m$ is defined as the minimal distance between two classes and hyperplane, designing optimal hyperplane is equal to maximizing $m$. If each of two classes has label +1 and -1 , $m$ can be expressed with the norm of $\mathbf{W}$ as illustrated in Fig. 6 and equation (11).

$$
m=\frac{2}{\|\mathbf{w}\|}
$$

SVM training can be explained by constrained optimization problem [23]. A data set $\left\{\mathbf{x}_{1}, \ldots, \mathbf{x}_{\mathbf{n}}\right\}$ and class label $y_{i} \in\{-1,1\}$ for $\mathbf{x}_{\mathbf{i}}$ are known, decision boundary must satisfy $y_{i}\left(\mathbf{w} \mathbf{x}_{\mathbf{i}}+b\right) \geq 0$ for all data. Maximizing $m$ is equal to minimizing the norm of $\mathbf{w}$ as deduced from equation (11). Therefore, SVM training is the same as solving constrained optimization problem, equation (12). 

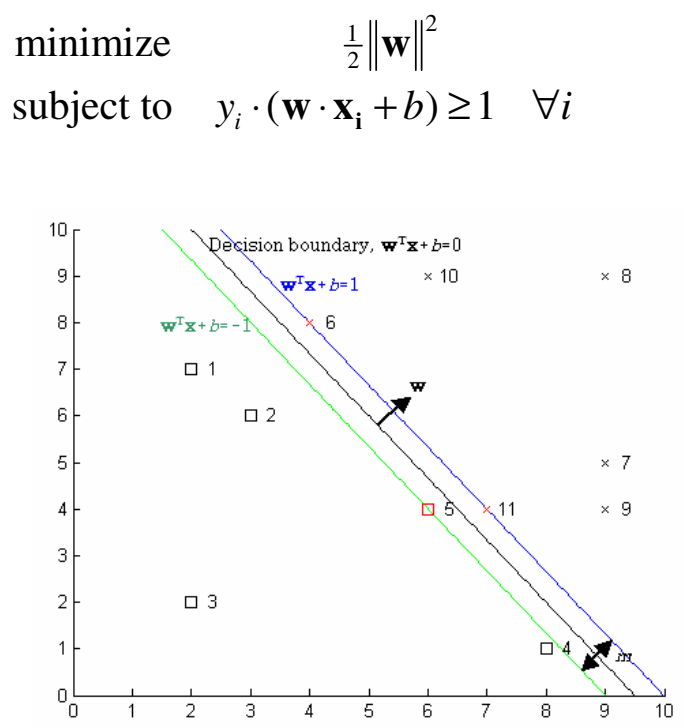

Fig. 6. Separation margin $m$ and decision boundary

An optimization problem with multiple inequality constraints can be solved using Lagrange multiplier. If data $\mathbf{x}_{\mathbf{i}}$, label $y_{i}$ are known, SVM training is the same as solving constrained optimization problem with respect to $W(\alpha)$ as in equation (13). Here, $\alpha$ is Lagrangian coefficient vector for every constraint. Since the problem is a type of $\mathrm{QP}(\mathrm{Quadratic}$ Programming) problem, various QP problem tools can be used. Once $\alpha$ is derived, $\mathbf{w}$ can be derived by equation (14). Therefore, $b$ can be derived by utilizing data on the boundary. Because only data on decision boundary, i.e. support vector, have non-zero coefficients and contribute to boundary equation, decision function can be expressed only by support vectors. Nonlinear decision boundary can be learned by introducing kernel function.

$$
\begin{aligned}
& \operatorname{maximize} W(\boldsymbol{\alpha})=\sum_{i=1}^{n} \alpha_{i}-\frac{1}{2} \sum_{i=1}^{n} \sum_{j=1}^{n} \alpha_{i} \alpha_{j} y_{i} y_{j} \mathbf{x}_{i}^{T} \mathbf{x}_{j} \\
& \text { subject to } \quad \alpha_{i} \geq 0, \quad \sum_{i=0}^{n} \alpha_{i} y_{i}=0 \\
& \mathbf{w}=\sum_{i=1}^{n} \alpha_{i} y_{i} \mathbf{x}_{i}
\end{aligned}
$$

The SVM of vehicle classifier is trained using 1,000 normalized images. $50 \%$ of the images are true images, and the others are false images. $648(=9 \times 24 \times 3)$ dimensional feature vector is acquired by applying 24 Gabor filters to 9 sub-windows and 
estimating 3 statistic values. Used kernel is 1st-order linear function. The learning result using SVMlight [24] is shown in Table 1.

Table 1. Vehicle classifier learning result

\begin{tabular}{|l|l|}
\hline Misclassified image & $38 / 1000$ \\
\hline Correct classification rate & $96.2 \%$ \\
\hline False positive, P(Vehicle I Other) & $5.6 \%$ \\
\hline False negative, P(Other I Vehicle) & $2 \%$ \\
\hline
\end{tabular}

The SVM of pedestrian classifier is trained using 1,200 normalized images. 700 of the normalized images are true images, and the others are false images. Used kernel is radial basis function. The learning result using SVMlight is shown in Table 2. It is noticeable that the system is able to recognize pedestrians of various sizes, colors and poses. Obstacles with various shapes and candidates from laser noise are correctly recognized as non-pedestrian images.

Table 2. Pedestrian classifier learning result

\begin{tabular}{|l|l|}
\hline Misclassified image & $63 / 1200$ \\
\hline Correct classification rate & $94.75 \%$ \\
\hline False positive, $\mathrm{P}($ Pedestrian I Other $)$ & $8.8 \%$ \\
\hline False negative, $\mathrm{P}($ Other I Pedestrian $)$ & $2.7 \%$ \\
\hline
\end{tabular}

\section{Obstacle Judgment}

Each cluster of range data creates a 'vehicle' candidate image and a 'pedestrian' candidate image. Vehicle classifier and pedestrian classifier verify the images, respectively. Generally, one of two classifiers presents positive response or both classifier present negative responses. However, if both classifiers present positive responses, the system recognizes the candidate as pedestrian in order to be conservative. The correlation between classifier outputs and obstacle judgment is shown in Table 3.

Table 3. The correlation between classifier outputs and final decision

\begin{tabular}{|c|c|c|}
\hline Pedestrian Classifier Output & Other & Pedestrian \\
\hline Vehicle Classifier Output & & \\
\hline Other & Other & Pedestrian \\
\hline Vehicle & Vehicle & Pedestrian \\
\hline
\end{tabular}

\section{Experiment Result}

406 images that are not used for vehicle classifier learning are used to test performance of vehicle classifier. $50 \%$ of the images are 'vehicle' image, and the others are 'other' 
images. The test result shows $95.07 \%$ of proper image recognition rate. Table 4 shows the test result of vehicle classifier. Fig. 7 shows correctly recognized candidate images. It is noticeable that various kinds of vehicles in different pose and size are successfully recognized.

Table 4. Test result of vehicle classifier

\begin{tabular}{|l|l|}
\hline Misclassified image & $20 / 406$ \\
\hline Correct classification rate & $95.07 \%$ \\
\hline False positive, P(Vehicle I Other) & $5.4 \%$ \\
\hline False negative, P(Other I Vehicle) & $5.4 \%$ \\
\hline
\end{tabular}



(a) Correctly recognized vehicle images



(b) Correctly recognized other images

Fig. 7. Candidate images correctly recognized by vehicle classifier

392 test images that are not used for pedestrian classifier learning are used to test performance of pedestrian classifier. $50 \%$ of the images are 'pedestrian' image, and the others are 'other' images. The test result shows $89.29 \%$ of proper image recognition rate. Table 5 shows the test result of pedestrian classifier. It is noticeable that correctly recognized candidate images include street crossing pose and street following pose.

Although developed recognition system successfully classifies vehicle and pedestrian in simple situations such as test track and inactive straight roadway, it fails to recognize in cluttered urban roadway because of complicated roadside objects. In such cases, clustering of range data becomes too noisy and leads to incorrect extraction of candidate image. These problems should be solved in the future.

Table 5. Test result of pedestrian classifier

\begin{tabular}{|l|l|}
\hline Misclassified image & $42 / 396$ \\
\hline Correct classification rate & $89.29 \%$ \\
\hline False positive, P(Pedestrian I Other) & $12.7 \%$ \\
\hline False negative, P(Other I Pedestrian) & $8.7 \%$ \\
\hline
\end{tabular}




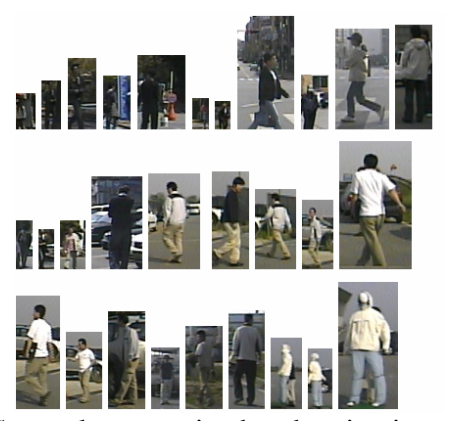

(a) Correctly recognized pedestrian images

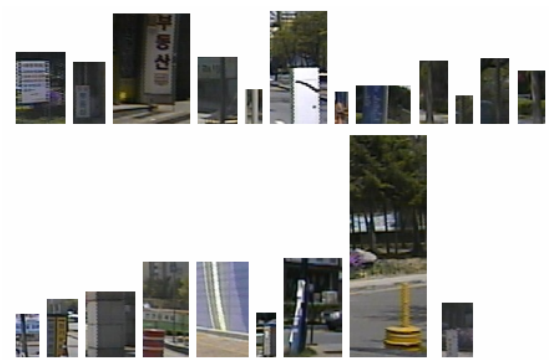

(b) Correctly recognized other images

Fig. 8. Candidate images correctly recognized by pedestrian classifier

\section{Conclusion}

This paper proposes a sensor fusion based obstacle detection and classification system. Obstacle's class information can be used for situation adaptive control system. Furthermore, it can reduce annoying false alarm rate of active pedestrian protection system such that users accept the new function as a practical daily tool. Experiments show that the same architecture can be applied for vehicle detection and pedestrian detection. Used Gabor filter bank and SVM are supposed to be suitable for hardware implementation. Our future works are improvement of range data clustering algorithm and implementation of system with FPGA to meet real-time performance.

\section{References}

1. Ardalan Vahidi and Azim Eskandarian, "Research Advances in Intelligent Collision Avoidance and Adaptive Cruise Control", IEEE Transaction on Intelligent Transportation Systems, Vol. 4, No. 3, Sep. 2003, pages: 143 153.

2. Akira Hattori, et al., "Development of Forward Collision Warning System Using the Driver Behavioral Information", SAE Paper No.: 2006-01-1462, The Society of Automotive Engineers, 2006.

3. Thierry Bellet, et al., "Driver behaviour analysis and adequacy judgement for manmachine cooperation: an application to anticollision", $5^{\text {th }}$ European Congress and Exhibition on Intelligent Transportation Systems and Services, 1 3 June 2005.

4. Meinecke, et al., "SAVE-U: First Experiences with a Pre-Crash System for Enhancing Pedestrian Safety", $5^{\text {th }}$ European Congress and Exhibition on Intelligent Transportation Systems and Services, 1 3 June 2005.

5. Takayuki Tsuji, Hideki Hashimoto, Nobuharu Nagaoka, "Intelligent Night Vision SystemNighttime Pedestrian Detection Assistance System", $12^{\text {th }}$ World Congress on Intelligent Transport Systems, 6 10 November 2005.

6. Zehang Sun, George Bebis, and Ronald Miller, "On-Road Vehicle Detection: A Review", IEEE Transaction on Pattern Analysis and Machine Intelligence, Vol. 28, No. 5, May 2006, pages: $1 \sim 18$. 
7. Zehang Sun, George Bebis, and Ronald Miller, "On-Road Vehicle Detection Using Evolutionary Gabor Filter Optimization”, IEEE Transaction on Intelligent Transportation Systems, Vol. 6, No. 2, June 2005, pages: 125 137.

8. Dariu M. Gavrila, "Sensor-Based Pedestrian Protection”, IEEE Intelligent Systems, Vol. 16, Issue 6, Nov.-Dec. 2001, pages: 77-81.

9. Kay Ch. Fuerstenberg, and Jochen Scholz, "Reliable Pedestrian Protection Using Laserscanners", 2005 IEEE Intelligent Vehicles Symposium, 6-8 June 2005, Pages: 142-146.

10. Florian Fölster, Hermann Rohling, and Marc-Michael Meinecke, "Pedestrian Recognition based on automotive radar sensors", 5th European Congress and Exhibition on ITS and Services, 1-3 June 2005.

11. Cristóbal Curio, et al., "Walking Pedestrian Recognition”, IEEE Transaction on Intelligent Transportation Systems, Vol. 1, No. 3, September 2000, pages: 155-163.

12. D.M. Gavrila, "Pedestrian Detection from a Moving Vehicle", LNCS 1843 (ECCV 2000), 2000, pages: 37-49.

13. A. Broggi, M. Bertozzi, A. Fascioli, and M. Sechi, "Shape-based Pedestrian Detection", IEEE Intelligent Vehicles Symposium 2000, October 3-5, 2000, pages:215-220.

14. Liang Zhao, and Charles E. Thorpe, "Stereo- and Neural Network-Based Pedestrian Detection", IEEE Transaction on Intelligent Transportation Systems, Vol. 1, No. 3, September 2000, pages: 148-154.

15. Grant Grubb, et al., "3D Vision Sensing for Improved Pedestrian Safety”, IEEE Intelligent Vehicles Symposium 2004, June 14-17, 2004, pages: 19-24.

16. Anuj Mohan, Constantine Papageorgiou, and Tomaso Poggio, "Example-Based Object Detection in Images by Components", IEEE Transaction on Pattern Analysis and Machine Intelligence, Vol. 23, No. 4, April 2001, pages:349-361.

17. Constantine Papageorgiou and Tomas Poggio, "A Trainable System for Object Detection", International Journal of Computer Vision, 38(1), 15-33, 2000, pages: 15-33.

18. Milch, S., Behrens, M., "Pedestrian Detection with Radar and Computer Vision", http://www.smart-microwave-sensors.de/html/publications.html, 2006.

19. L. Walchshäusl, et al., "Detection of Road Users in Fused Sensor Data Streams for Collision Mitigation", $10^{\text {th }}$ International Forum on Advanced Microsystems and Automotive Applications (AMAA 2006), Berlin, Germany, April 25-26, 2006.

20. Javier R. Movellan, "Tutorial on Gabor Filters", http://mplab.ucsd.edu/tutorials/pdfs/gabor.pdf, 2006.

21. B. S. Manjunath and W. Y. Ma, "Texture Features for Browsing and Retrieval of Image Data”, IEEE Transaction on Pattern Analysis and Machine Intelligence, Vol. 18, No. 8, August 1996, pages: 837-842.

22. Christopher J. C. Burges, "A Tutorial on Support Vector Machines for Pattern Recognition", Data Mining and Knowledge Discovery, 2, 121-167 (1998).

23. Martin Law, "A Simple Introduction to Support Vector Machines", http://www.cse.msu.edu/ lawhiu/intro_SVM_new.ppt, 2006.

24. Thorsten Joachims, "SVMlight: Support Vector Machine", http://svmlight.joachims.org, 2006. 